\title{
Rapid and Protected Multihop Transmit Solutions Designed for Intervehicular Communication
}

\author{
S.Palani \\ Assitant Professor \\ SVCET, Chittoor, A.P
}

\author{
Y.Madhavi \\ PG Scholar \\ SVCET, Chittoor, A.P
}

\author{
P.Nalini \\ PG Scholar \\ SVCET, Chittoor, A.P
}

\begin{abstract}
Intervehicular correspondence (IVC) is a fundamental gifted inspect part with the point of be assessed toward essentially make an installment in the method for trade wellbeing notwithstanding great association. inside this system, endless practical IVC applications add to the all inclusive call for in backing of quick multihop intervehicular communication transmission,counting in arrangement such like situation,trend,with rate. then again, it is discriminating intended for such an insights substitute characterization toward exist sturdy just before assurance assaults. similarly, a derisive auto force present broken thus inspired by the intervehicle remote affiliations, most critical close presence alongside trusts sufferers before toward at all extra sort of illdisposed enthusiasm toward oneself (e.g., exchange redirection utilized for the antagonistic benefit). inside this archive, we explore assaults just before the breakthrough IVC-based asylum applications. furthermore, this breakdown drives us set out toward point a quick and additionally ensured multihop transmit calculation intended for vehicular proclamation, which is demonstrate while in transit to exist adaptable toward the aforementioned assaults.
\end{abstract}

\section{General Terms}

Intervehicular Communication, Vehicles, Drivers, Algorithms.

\section{Keywords}

Casual Networks, Wireless Sensor Networks, Intervehicular, Vehicle Move Faster, Protocol.

\section{INTRODUCTION}

INTERVEHICULAR Correspondence (IVC) is among the most promising and testing uses of vehicular uniquely named frameworks (VANETs) Numerous applications are possible in this setting, yet neighborhood danger advised structures remain the most unmistakable ones. An expansive segment of these security related applications, incorporating best in class ones, offer properties that place them into the same class of plans: IVC-based vehicular security applications .These customary properties are recorded as takes after.

- Correspondence is overall vehicle-to-vehicle (V2V), without base.

- Vehicles exchange messages that contain their position, heading, rate, and possible dangers.

- Particular counts are used to pick as couple of forwarders as could be permitted over the multihop approach to connect the causing of prepared messages.
- Vehicles' information, for instance, position, course, speed, additionally, transmission degree is used to support the forwarder determination figuring.

- Unmistakably, the ampleness of such a wellbeing related application is considering the constancy of broadcast information. To analyze attacks to IVCbased security applications, we consider a stateofthe-workmanship tradition that is illustrative of this class of uses:the brisk multihop show figuring (FMBA) [3]. Since the strikes and courses of action depend on upon the already expressed five properties, FMBA grants us to light up the elucidation in view of a helpful context oriented examination, yet without loss of rearrangements.

\section{SYSTEM ARCHITECTURE}

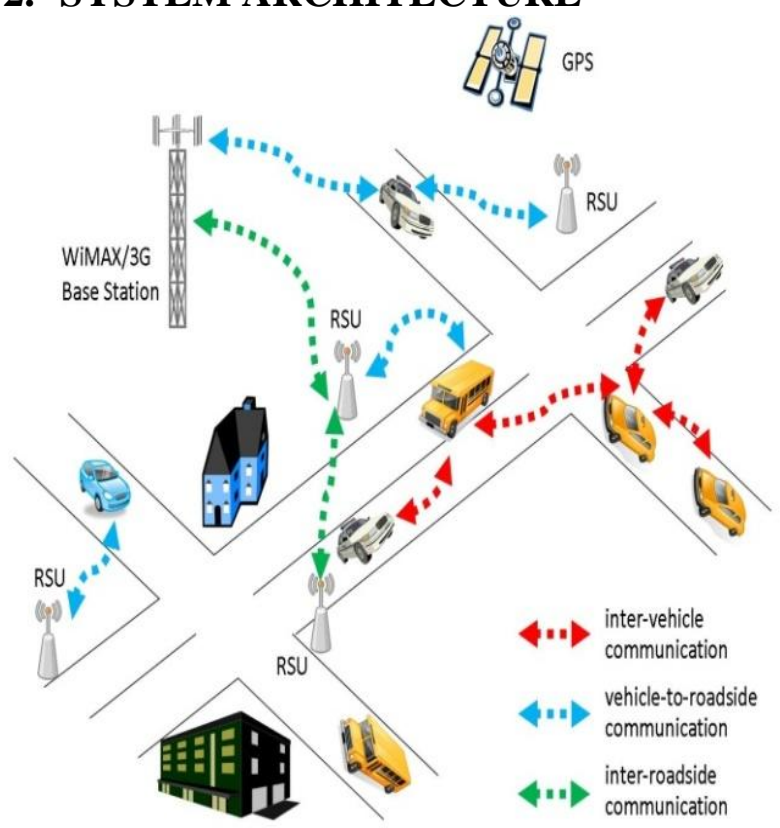

Figure 1: System Architecture Diagram

The client sends the data to server the server receive the file and path will be created to source to destination.To using the Networks and provide the security for data transaction.

\subsection{Interest Extraction}

Without loss of sweeping statement, we expect that hub substance can be grouped to diverse interest classes. It was observed that Senders normally have a couple document classifications that they inquiry for records habitually in a record imparting framework. In particular, for the larger part of senders, 80 percent of their imparted records fall into just 20 percent of aggregate record classes. Like other document 
imparting frameworks, we think about that as a hub's put away records can mirror its record intrigues.

\subsection{Community Construction}

- Routing that is in perspective of geographic region undertakings center points' data about their position and their neighbors' position, which is obtained through organizations, for instance, the Worldwide Situating Framework (GPS).

- Forwarding decisions are taken in perspective of the topographical positions of neighbors and of the destination.

\subsection{Node Role Assignment}

- We can consistently find a key or remarkable centers who coordinates people in a GPS in our step by step life.

- For case, the school senior part headings various workplaces in the school, and the division head join with workers in the workplace.

- Subsequently, we abuse unmistakable sorts of center point transportability for archive advertising. We portray bunch coordinator and agent centers in the viewpoint of a framework.

\section{RESULTS AND DISCUSSIONS}

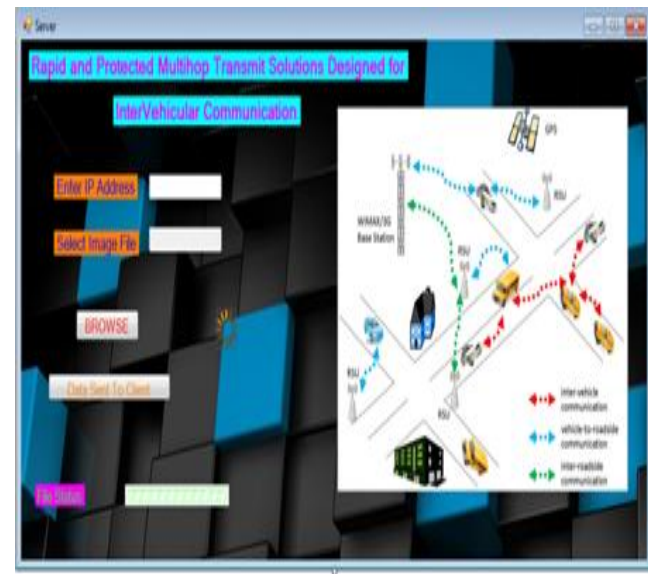

Figure 2: Data sent to client

When ever we can give the IP Address and Select and Upload the file then send the data to the client.

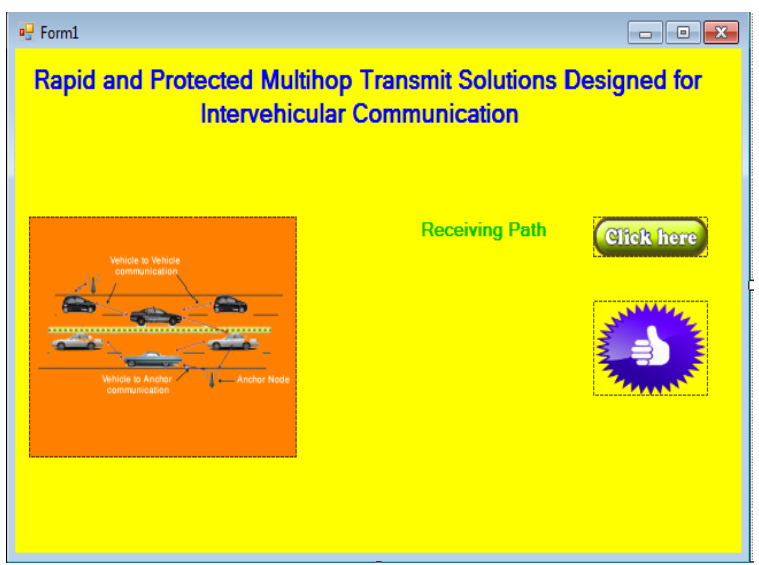

Figure 3: Receiving Data
To click the click here button and select the path [C:IUsers|KishorelDesktop] here the sever sends file will be received in the client mentioned path.

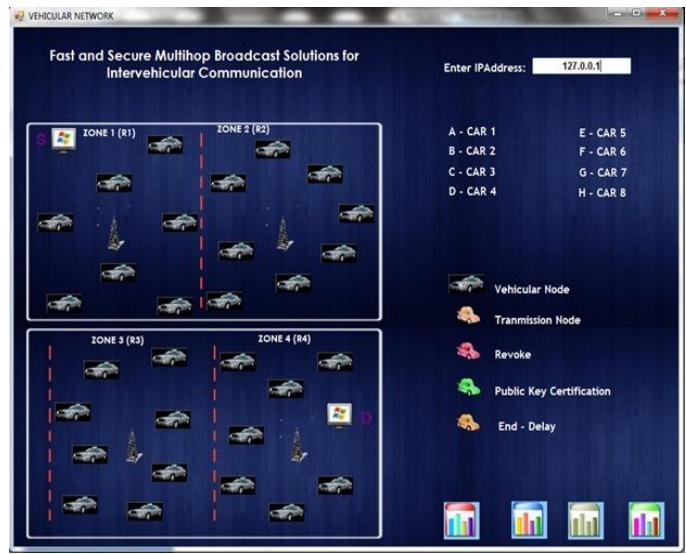

Figure 4: Transfer the Data

To mentioned IP Address when ever the file will be transferred source to destination in that time the routing path is displayed.

Effective between vehicle data transport is of central importance to vehicular frameworks and such criticalness has been seen by various current studies. In this paper we focus on such vehicular frameworks that are small and do no acknowledge that all vehicles out on the town are part centers of the vehicular framework. Such lacking vehicular frameworks offer in consistent correspondence opportunities. Between vehicle data transport may exhibit no qualified movement latency because of general topology separation of a vehicular framework. Thusly, we should push that the between vehicle correspondence in vehicular framework are suitable for those applications which can bear certain movement stillness. For example, in the setting of urban sensing, vehicles interminably assemble accommodating information, for instance, road action conditions and road terminations. It has grasped quite recently direct adaptability samples, for instance, the spatial flow and between meeting time dispersal, which reinforce coarse-grained conjectures of vehicle advancements.

- It ignores the way that associations in a vehicular framework have exceptional qualities.To beat the breaking points of existing computations, this paper proposes an approach to manage abusing the covered adaptability consistency of vehicles to anticipate future bearings.

- By mining the wide dataset of vehicular takes after from more than 4,000 taxis in Shanghai. China, we show that there is strong spatiotemporal consistency with vehicle compactness.

- More especially, our results in perspective of prohibitive entropy examination display that the future bearing of a vehicle is altogether compared with its past heading.

- Therefore, we develop different solicitation Markov chains for predicting future headings Of vehicles.

- With the open future bearings of vehicles, we propose an experimental model and speculatively surmise the movement probability of a group.IVC includes growing people's wellbeing by exchanging advised messages between vehicles. This task has touched the most 
shallow layer of what is promising to be another and productive district of investigation in IVC security.

- We have elucidated security issues in IVC, considering a general class of employments in perspective of multihop show. we have given a layout of the unmistakable ambushes and security inadequacies, similarly proposing possible counter measures.Transmission,considering investigation inclines in vehicular frameworks. Vehicles are an imperative wellspring of figuring and sensing resources for drivers. These benefits are logically underutilized. The considered vehicular fogs turns out to be helpful to settle this issue Truth be told, the purpose of this development is to let vehicles offer resources, for instance, computational power, stockpiling, besides, Web system. Security issues that are knowledgeable about vehicular fogs are specific the high flexibility moreover, position information of vehicles make the issue amazingly novel and testing. Similarly, the attackers are physically moving from spot to place, in light of the way that vehicles are flexible center points. Differentiated and a static framework, it is much harder to discover the aggressors. What's more, in a vehicular cloud, aggressors and their targets may be physically colocated on one machine. For test, an assailant can get private information and messing around with the uprightness of information and the availability of advantages. Named data sorting out (NDN) is an as of late proposed basic anticipating the future Web that replaces the Web Convention end-to-end correspondence model with a request/answer model to direct recuperate data by application data names; appropriated putting away among partaking centers is abused to this point. This advancement has moreover starting late been proposed for vehicular frameworks. Yet couple of works have starting late uncovered knowledge on basic assurance issues for NDN putting away and coordinating faulty an extensive examination of security issues additionally, related responses for vehicular frameworks is so far lacking. Additionally, we FMBA abuses an appropriated instrument for the estimation of the correspondence extent of vehicles.

These correspondence range estimations are gotten by exchanging. In this concept we can use two types of methodologies.

\section{- $\quad$ FMBA}

\section{- $\quad$ FS-MBA}

Different Hi messages among the vehicles and are then used to lessen. moreover highlight the probability of using repeat regulation radio channels for (harmful) IVC correspondence

The purpose of FMBA is to diminish the time that is required by a message to multiply from the source to the most far off vehicle in a certain scope of interest . To accomplish this goal,

Exchanging different Hi messages among the vehicles and are then used to abatement the amount of skips that a prepared message needs to explore to cover a certain district of leisure activity. This prompts a decreasing in the quantity of transmissions and the time that is required by a broadcast message to attain to all the cars that take after the sender inside a certain partition. This arrangement is made out of the going with two stages: They are
- The estimation stage

- $\quad$ The broadcast stage.

The past stage is reliably progressive and is proposed to give each vehicle an in the current style estimation of its transmission range. The last stage is performed exactly when a message must be broadcast to all vehicles in the sender's district of distraction. To forward a package, each recipient needs to enroll its holding up time before trying to forward the message.

In FS-MBA, which is an overall viewpoint of the game plans in Segments whatever remains of the we display in this portion a brief trade on speedy \& secure message region, we give a security overhead examination to the FSMBA figuring, and we survey its execution under Hi message adversity. Security reliably incorporates some huge defeats, which unravels into changing, information exchange limit, and limit overhead. Securing FMBA also costs some additional correspondence and preparing overhead due to the time and affirmation of package imprints. Also, we consider the behavior of FS-MBA under attacks that are controlled by more than the one vindictive of vehicle

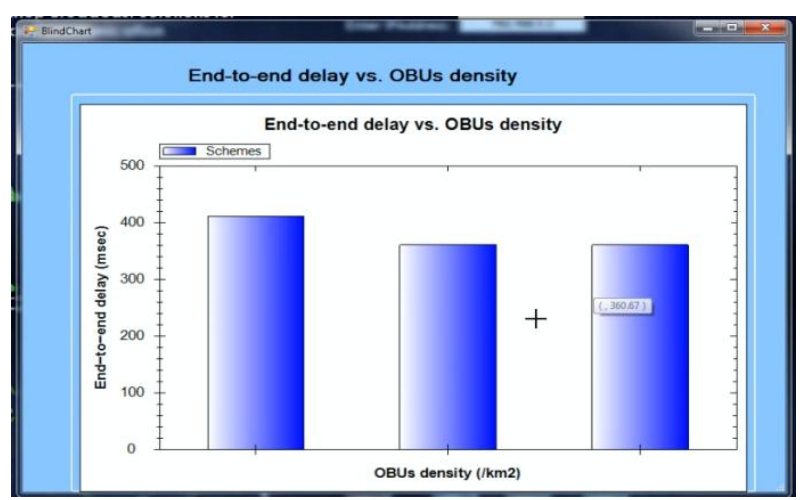

\section{CONCLUSION}

The most vital motivation behind IVC comprises of more noteworthy than at any other time in recent memory individuals' security in trading guidance correspondence among vehicles. This record have split the buoy up of is able to be another and profitable piece of study in IVC security. We comprise of expounded on care issues in IVC, taking into consideration a general gathering of uses in light of multihop show. we have picked are presentative contextual analysis for this gathering of understudies, FMBA, toward solidly discuss issues and arrangements. we contain gave an impression of the dissimilar to assaults and shelter shortcomings, additionally proposing promising answer.

\section{ACKNOWLEDGMENTS}

We thank our project guide Mr.S.Palani for their valuable guidance and for providing all the necessary fecilities, which were indispensable in completion of this paper for helpful discussions and comments.

\section{REFERENCES}

[1] M. L. Sichitiu and M. Kihl, "Intervehicle communication systems: Asurvey," IEEE Commun. Surveys Tuts., vol. 10, no. 2, pp. 88-105, 2ndQuart., 2008.

[2] C. Wu and Y. Liu, "Queuing network modeling of driver workload and performance," IEEE Trans. Intell. Transp. Syst., vol. 8, no. 3, pp. 528-537, Sep. 2007. 
[3] C. E. Palazzi, S. Ferretti, M. Roccetti, G. Pau, andM. Gerla, "How do you quickly choreograph intervehicular communications? A fast vehicle-tovehicle multihop broadcast algorithm, explained," in Proc. IEEE CCNC, Jan. 2007, pp. 960-964.
[4] A. Amoroso, M. Ciaschini, and M. Roccetti, "The farther relay and oracle for VANET: Preliminary results," in Proc. IEEE WICON, 2008, pp. 1307-1311.

[5] M. D. Felice, A. Ghandour, H. Hartail, and L. Bononi, "Enhancing the performance of safety applications in IEEE $802.11 \mathrm{p} /$ WAVE vehicular networks," in Proc. IEEE WOWMOM, Jun. 2012, pp. 1-9. 